\title{
The Impact of the FIFA 11+ Program on the Injury in Soccer Players: A Systematic Review
}

\author{
Hyunmin Kim¹, Juseoung Lee', Junghoon Kim ${ }^{1 *}$ \\ ' Department of Sports Medicine, Graduate School of Sports Convergence, Korea Maritime \& Ocean University, Busan, \\ Republic of Korea
}

Received: September 2, 2020

Accepted: October 15, 2020

Published online: October 31, 2020

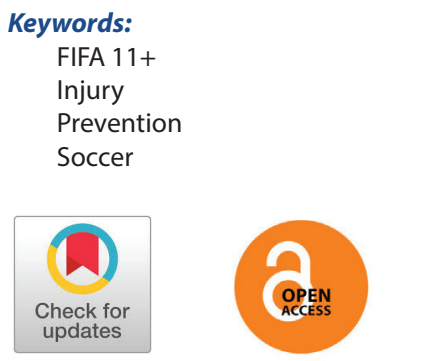

\section{ABSTRACT}

OBJECTIVES The purpose of this study was to integrate information about the effects of FIFA 11+ injury prevention program in soccer.

DESIGN AND METHODS Systematic review. We used PubMed, Medline, PMC, and Web of Science to search randomized controlled trials comparing FIFA $11+$ injury prevention program with control groups among soccer players.

RESULTS Total of 13 studies was selected through eligibility criteria. 7 studies included overall, ankle, knee, and hamstring injury rate between FIFA $11+$ versus control groups, while the other 6 studies included improvement of physical performance, such as vertical jump, $20 \mathrm{~m}$ sprint, agility, hip adduction strength, and FMS total score between FIFA 11+ versus control groups. There was a lower rate of injury in the intervention group from all 7 studies. Couple of studies had different criteria to compare with other studies. Most values of physical performance were also significantly improved from all 6 studies except for the values of agility time.

CONCLUSIONS Multiple studies have proven injury rate reduction and physical performance improvement of FIFA 11 + program between intervention and control groups. We also found that it at least required a few weeks of intervention time to be effective. Therefore, coaches and players might need to consider including FIFA $11+$ program as a warm-up period to prepare the regular season.

( ) The Asian Society of Kinesiology and the Korean Academy of Kinesiology

\section{Introduction}

Soccer is one of the most popular sports worldwide by far. According to Fédération Internationale de Football Association (FIFA), the soccer popularity now is close to 270 million all over the world [1]. Similar to other sports, soccer has also been causing injuries to all-aged group players. A recent study demonstrated that injury rate of soccer game was liable to rise aiming to all-aged group players, with an average occurrence of 15 to 20 injuries based on 1,000 hours

*Correspondence: Junghoon Kim, Ph.D., Sports and Exercise Medicine Laboratory, Korea Maritime \& Ocean University; 727 Taejong-ro, Yeongdo-Gu, Busan 49112 Republic of Korea; Phone: +82-51-410-4792; E-mail: junghoonkim@kmou.ac.kr of game-play among 15 or older players [2]. According to a cohort study written by Stubbe reported that Dutch Premier League soccer players were injured 32.8 times/1000h of game play and 2.8 times/1000h of training session[3]. $87 \%$ of the injuries occurred on lower extremities, $58 \%$ of which were caused by non-contact mechanism[3]. The most frequent injuries to collegiate soccer players were ankle sprains that occurred 3.19 times based on 1,000 exposures (game and training time). Thigh muscle sprains and knee sprains were second and third most frequent injuries, which occurred 2.28 times and 2.07 times in 1,000 exposures, respectively [4]. For these reasons, coaches and players might need to realize the 
severity and danger of risks that exists within its sport and search for the best way to protect themselves from injuries.

The injury prevention program called 'FIFA 11' was found by FIFA's Medical Assessment and Research Center (F-MARC) and it has been recognizing as a simple dynamic exercise program used before training and games for 15-20 minutes performed 2-3 times per week throughout the whole season [5-7]. Also, after addressing the deficiencies by adding up dynamic warm-up programs, it was renamed as 'FIFA 11+' composed with 15 exercises total. FIFA $11+$ program is divided into 3 important parts of exercises. First part starts with slow running and dynamic stretching exercises. Then, second part consists of core and lower extremity strengthening, plyometric and balance exercises such as, plank, Nordic Hamstring Exercise, Single-leg Stance, Lunge and Jumping Squat to increase motor capacity of individuals. Finally, high-speed running and cutting exercises follow at the end to warm them up before the actual game or training sessions [8]. What these exercises emphasize intensively is strengthening core and lower extremity muscles, improving coordination, balance, and the response of static and dynamic neuromuscular control [9]. The FIFA11+ program is designed as a warm-up that takes place before soccer training, with the aim of getting the proper skills and improving the neuromuscular coordination. This program has also been proven in both males and females including amateur and professional soccer leagues [6]. Although previous studies have been investigated the effectiveness of the FIFA11+ programs but have not consistently reported a statistically significant reduction in injury rate and improve physical performance from utilizing the program.

Therefore, in this systematic review, we aimed to investigate how effective FIFA $11+$ program is to reduce injury rate and improve physical performance for male soccer players by integrating the evidences from recent clinical studies. Information of injury rate reductions and physical performance improvements through FIFA 11+ injury prevention program will be included to demonstrate how much this warm-up protocol can help prevent soccer players from risk of injury by strengthening core and lower extremity muscle, improving coordination and balance, and responding neuromuscular control.

\section{Method}

\section{Database}

To identify the currently well-designed clinical research papers, a literature search was conducted using database including PubMed, Medline, PubMed Central, and Web of Science. We searched for studies published between 2015 and 2020. The search key words were "FIFA 11+", "soccer", and "injury" in the title or abstract. We only included our search to original research published in English language. Finally, we also limited the study subjects to male soccer players aged $\geq 16$, and the study design to 'randomized controlled trial' and 'cohort study'.

\section{Selection Criteria}

This review followed the recommendations of the preferred reporting items for systematic review (PRISMA) statement. $<$ Figure $1>$ shown the included details of the selection of trails according to the review guideline from PRISMA. Firstly, we found 94 articles using the search keywords, and found 13 duplicated articles from each data base. We conducted primary review the title and abstract for remained 81 articles. Then, 61 articles were removed after the primary review due to not meeting criteria. Removed articles had different age,

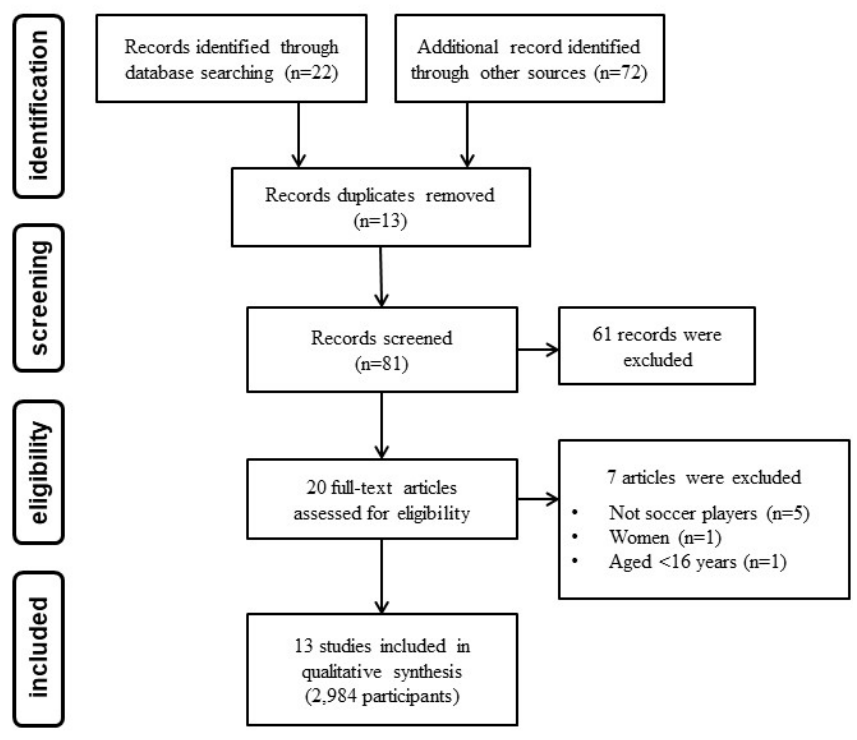

Figure 1. Flowchart of the selection of trials included in this systematic review. 
gender or sports athletes group of participants or were not RCT studies. Finally, 7 articles were excluded out of 20 articles being assessed. Details of the extraction process are listed on $<$ Figure $1>$.

\section{Results}

Out of 13 studies, 7 of them determined overall, ankle, knee, and hamstring injury rate between FIFA 11+ versus control groups. Total of 2772 soccer players from 3 different countries participated in the studies investigating injury rate reductions. The study characteristics of included studies for overall, ankle, knee, and hamstring injuries are presented in $<$ Table $1>$.

Data summarization of overall, ankle, knee and hamstring injury rate among included studies is presented in $<$ Table
$2>$. All studies showed lower injury rate in the intervention group. Most studies except for one study written by Chena et al. presented injury rate within 1,000 hours [10]. Chena and colleagues had investigated players for 2 consecutive seasons divided intervention and control group by each season. In case of Whalen et al., both intervention and control group conducted FIFA $11+$ program and the intervention group performed part 2 of the program again after game or training was done. Therefore, we decided to put both values in the intervention group [11].

The other 6 studies determined improvement of physical performance, such as vertical jump, 20m sprint, agility, hip adduction strength, and FMS total score between FIFA 11+ versus control group. Total of 212 soccer players from 4 different countries participated in the studies investigating physical performance improvements. The study characteristics

Table 1. Study characteristics summarization of the included studies for injury rates

\begin{tabular}{|c|c|c|c|c|c|c|c|c|c|}
\hline Study & Region & Age & Design & Level & Duration & $\begin{array}{l}\text { Type of exercise in } \\
\text { intervention group }\end{array}$ & $\begin{array}{l}\text { Type of Exercise } \\
\text { in Control Group }\end{array}$ & $\begin{array}{l}\text { Frequency/ } \\
\text { week }\end{array}$ & Outcome \\
\hline $\begin{array}{l}\text { Granelli, } \\
\text { et al. } \\
(2015)\end{array}$ & $\begin{array}{l}\text { United } \\
\text { States }\end{array}$ & $18-25$ & $\begin{array}{l}\text { Cluster- } \\
\text { Randomized } \\
\text { Controlled } \\
\text { Trial }\end{array}$ & $\begin{array}{l}\text { Collegiate } \\
\text { Soccer }\end{array}$ & 6 months & FIFA $11+$ & Usual & 3 & $\begin{array}{l}\text { Overall Injury } \\
\text { Ankle Injury } \\
\text { Knee Injury } \\
\text { Hamstring Injury }\end{array}$ \\
\hline $\begin{array}{l}\text { Attar, et } \\
\text { al. }(2017)\end{array}$ & Australia & $16-18$ & $\begin{array}{l}\text { Cluster- } \\
\text { Randomized } \\
\text { Trial }\end{array}$ & $\begin{array}{l}\text { Amateur } \\
\text { league }\end{array}$ & 6 months & $\begin{array}{l}\text { Pre \&Post training } \\
\text { FIFA } 11+\end{array}$ & $\begin{array}{l}\text { Pre-training FIFA } \\
11+\end{array}$ & $2-3$ & $\begin{array}{l}\text { Overall Injury } \\
\text { Ankle Injury } \\
\text { Knee Injury } \\
\text { Hamstring Injury }\end{array}$ \\
\hline $\begin{array}{l}\text { Garcia, et } \\
\text { al. (2017) }\end{array}$ & Spain & 24.7 & Cohort Study & $\begin{array}{l}\text { Amateur } \\
\text { league }\end{array}$ & 4 seasons & FIFA $11+$ & Usual & 3 & $\begin{array}{l}\text { Hamstring Injury } \\
\text { Ankle Injury } \\
\text { (LAL Injury) }\end{array}$ \\
\hline $\begin{array}{l}\text { Granelli, } \\
\text { et al. } \\
\text { (2017) }\end{array}$ & $\begin{array}{l}\text { United } \\
\text { States }\end{array}$ & $18-25$ & $\begin{array}{l}\text { Cluster- } \\
\text { Randomized } \\
\text { Controlled } \\
\text { Trial }\end{array}$ & $\begin{array}{l}\text { Collegiate } \\
\text { Soccer }\end{array}$ & 6 months & FIFA $11+$ & Usual & 3 & $\begin{array}{l}\text { Knee Injury } \\
\text { (ACL Injury) }\end{array}$ \\
\hline $\begin{array}{l}\text { Granelli, } \\
\text { et al. } \\
\text { (2018) }\end{array}$ & $\begin{array}{l}\text { United } \\
\text { States }\end{array}$ & $18-25$ & $\begin{array}{l}\text { Cluster- } \\
\text { Randomized } \\
\text { Controlled } \\
\text { Trial }\end{array}$ & $\begin{array}{l}\text { Collegiate } \\
\text { Soccer }\end{array}$ & 6 months & FIFA $11+$ & Usual & 3 & $\begin{array}{l}\text { Overall Injury } \\
\text { Ankle Injury } \\
\text { Knee Injury } \\
\text { Hamstring Injury }\end{array}$ \\
\hline $\begin{array}{l}\text { Whalan, } \\
\text { et al. } \\
\text { (2019) }\end{array}$ & Australia & 24.3 & $\begin{array}{l}\text { Cluster- } \\
\text { Randomized } \\
\text { Trial }\end{array}$ & $\begin{array}{l}\text { Semi- } \\
\text { professional } \\
\text { league }\end{array}$ & 6 months & Part 2 post training & $\begin{array}{l}\text { Standard FIFA } \\
11+\end{array}$ & 2 & $\begin{array}{l}\text { Overall Injury } \\
\text { Ankle Injury } \\
\text { Knee Injury } \\
\text { Hamstring Injury }\end{array}$ \\
\hline $\begin{array}{l}\text { Chena, et } \\
\text { al. (2019) }\end{array}$ & Spain & $16-23$ & Cohort Study & $\begin{array}{l}\text { Amateur } \\
\text { league }\end{array}$ & $\begin{array}{l}12 \\
\text { months }\end{array}$ & FIFA $11+$ & Usual & 3 & $\begin{array}{l}\text { Overall Injury } \\
\text { Ankle Injury } \\
\text { Knee Injury } \\
\text { Hamstring Injury }\end{array}$ \\
\hline
\end{tabular}

LAL: Lateral Ankle Ligament, ACL: Anterior Cruciate Ligament 
of included studies for physical performance improvements are presented in $<$ Table $3>$.

Data summarization of physical performance among included studies is presented in <Table $4>$. Most values of physical performance were significantly improved; however, results of agility time(s) showed non-significant between prepost test in every section for 2 studies written by Ayala et al. [12] and Taher \& Parnow [13].

Table 2. Injury rates of overall, ankle, knee, and hamstring injuries among the included studies

\begin{tabular}{|c|c|c|c|c|c|c|c|c|}
\hline \multirow{3}{*}{ Study } & \multicolumn{8}{|c|}{ IR per 1000 Aes (Athlete-exposures) } \\
\hline & \multicolumn{2}{|c|}{ Overall Injury } & \multicolumn{2}{|c|}{ Ankle Injury } & \multicolumn{2}{|c|}{ Knee Injury } & \multicolumn{2}{|c|}{ Hamstring Injury } \\
\hline & Intervention group & Control group & Intervention group & Control group & Intervention group & Control group & Intervention group & Control group \\
\hline \multicolumn{9}{|l|}{ Granelli, et al. (2015) } \\
\hline Granelli, et al (2017) & 8.09 & 15.04 & 1.675 & 2.6 & 0.97 & 2.3 & 0.45 & 1.24 \\
\hline \multicolumn{9}{|l|}{ Granelli, et al (2018) } \\
\hline Attar, et al. (2017) & 0.726 & 2.593 & 0.11 & 0.38 & 0.11 & 0.41 & 0.06 & 0.28 \\
\hline Garcia, et al. (2017) & Not provided & $\begin{array}{c}\text { Not } \\
\text { provided }\end{array}$ & 1.88 & 3.14 & Not provided & $\begin{array}{l}\text { Not } \\
\text { provided }\end{array}$ & 2.26 & 4.39 \\
\hline \multirow[t]{2}{*}{ Whalan, et al. (2019) ${ }^{a}$} & $\begin{array}{c}12.3 \\
\text { (standard 11+) }\end{array}$ & \multirow{2}{*}{$\begin{array}{c}\text { Not } \\
\text { provided }\end{array}$} & $\begin{array}{c}2.1 \\
\text { (standard 11+) }\end{array}$ & \multirow{2}{*}{$\begin{array}{l}\text { Not } \\
\text { provided }\end{array}$} & $\begin{array}{c}2.3 \\
\text { (standard 11+) }\end{array}$ & \multirow{2}{*}{$\begin{array}{l}\text { Not } \\
\text { provided }\end{array}$} & $\begin{array}{c}2.0 \\
\text { (standard 11+) }\end{array}$ & \multirow{2}{*}{$\begin{array}{c}\text { Not } \\
\text { provided }\end{array}$} \\
\hline & 11.8 ( $\mathrm{P} 2$ post) & & 1.4 (P2 post) & & 2.1 (P2 post) & & 1.6 (P2 post) & \\
\hline Chena, et al. (2019) ${ }^{b}$ & $\begin{array}{c}0.27 \\
\text { (Intervention } \\
\text { Season) }\end{array}$ & $\begin{array}{c}0.73 \\
\text { (Control } \\
\text { Season) }\end{array}$ & $\begin{array}{l}17 \text { injuries/ } \\
\text { season }\end{array}$ & $\begin{array}{l}44 \text { injuries/ } \\
\text { season }\end{array}$ & $\begin{array}{l}10 \text { injuries/ } \\
\text { season }\end{array}$ & $\begin{array}{l}18 \text { injuries/ } \\
\text { season }\end{array}$ & $\begin{array}{l}5 \text { injuries/ } \\
\text { season }\end{array}$ & $\begin{array}{l}23 \text { injuries/ } \\
\text { season }\end{array}$ \\
\hline
\end{tabular}

Table 3. Study characteristics summarization of the included studies for performance improvements

\begin{tabular}{|c|c|c|c|c|c|c|c|c|c|}
\hline Study & Region & Age & Design & Level & Duration & $\begin{array}{l}\text { Type of exercise in } \\
\text { intervention group }\end{array}$ & $\begin{array}{l}\text { Type of Exercise } \\
\text { in Control Group }\end{array}$ & $\begin{array}{l}\text { Frequency/ } \\
\text { week }\end{array}$ & Outcome \\
\hline $\begin{array}{l}\text { Ayala, et al. } \\
(2016)\end{array}$ & Spain & $16-18$ & $\begin{array}{l}\text { Randomized- } \\
\text { Controlled Trial }\end{array}$ & $\begin{array}{l}\text { Amateur } \\
\text { league }\end{array}$ & 4 weeks & FIFA $11+$ & Usual & 3 & $\begin{array}{l}\text { Vertical jump, } \\
\text { Agility }\end{array}$ \\
\hline $\begin{array}{l}\text { Akbari, et } \\
\text { al. }(2018)\end{array}$ & Iran & $16-19$ & $\begin{array}{l}\text { Randomized- } \\
\text { Controlled Trial }\end{array}$ & $\begin{array}{l}\text { Elite } \\
\text { soccer }\end{array}$ & 8 weeks & FIFA 11+ & Usual & 3 & Vertical jump \\
\hline $\begin{array}{l}\text { Harøy } \\
(2017)\end{array}$ & Norway & $16-19$ & $\begin{array}{l}\text { Randomized- } \\
\text { Controlled Trial }\end{array}$ & $\begin{array}{l}\text { Elite } \\
\text { soccer }\end{array}$ & 8 weeks & $\begin{array}{l}\text { FIFA } 11+ \\
\text { (Copenhagen } \\
\text { adduction exercise) }\end{array}$ & $\begin{array}{l}\text { FIFA } 11+\text { (Nordic } \\
\text { hamstring } \\
\text { exercise) }\end{array}$ & 3 & $\begin{array}{l}\text { Eccentric hip } \\
\text { adduction } \\
\text { strength }\end{array}$ \\
\hline $\begin{array}{l}\text { Taher \& } \\
\text { Parnow } \\
(2017)\end{array}$ & Iran & $23 \pm 4$ & $\begin{array}{l}\text { Randomized- } \\
\text { Controlled Trial }\end{array}$ & $\begin{array}{l}\text { Collegiate } \\
\text { soccer }\end{array}$ & 1 day & $\begin{array}{l}\text { FIFA } 11+\text {, Static, } \\
\text { Dynamic Stretch }\end{array}$ & $\begin{array}{l}\text { FIFA } 11+, \text { Static, } \\
\text { Dynamic Stretch }\end{array}$ & 1 & $\begin{array}{l}\text { Vertical Jump, } \\
20 \mathrm{~m} \text { sprint, } \\
\text { Agility, Knee } \\
\text { flexibility }\end{array}$ \\
\hline $\begin{array}{l}\text { Nawed, et } \\
\text { al. (2018) }\end{array}$ & India & $20-23$ & $\begin{array}{l}\text { Cluster- } \\
\text { Randomized } \\
\text { Trial }\end{array}$ & $\begin{array}{l}\text { Amateur } \\
\text { league }\end{array}$ & 12 weeks & FIFA $11+$ & Usual & 5 & $\begin{array}{l}\text { Vertical jump } \\
20 \mathrm{~m} \text { sprint }\end{array}$ \\
\hline $\begin{array}{l}\text { Rey, et al } \\
(2018)\end{array}$ & Spain & $24.7 \pm 3.8$ & $\begin{array}{l}\text { Randomized- } \\
\text { Controlled Trial }\end{array}$ & $\begin{array}{l}\text { Amateur } \\
\text { soccer }\end{array}$ & 6 weeks & FIFA $11+$ & Usual & 3 & FMS \\
\hline
\end{tabular}

FMS: Functional Movement Screen 
Table 4. Performance improvements of VJ, $20 \mathrm{~m}$ sprint, agility, hip adduction strength, and FMS score among the included studies

\begin{tabular}{|c|c|c|c|c|c|c|}
\hline \multirow{2}{*}{ Study } & \multicolumn{3}{|c|}{ Intervention group } & \multicolumn{3}{|c|}{ Control Group } \\
\hline & Pre & Post & P-value & Pre & Post & P-value \\
\hline \multicolumn{7}{|l|}{ Vertical Jump (cm) } \\
\hline Ayala, et al. (2016) & $26.7 \pm 2.2$ & $26.6 \pm 3.5$ & $<0.05$ & $24.1 \pm 2.2$ & $22.4 \pm 5.5$ & $<0.05$ \\
\hline Akbari, et al. (2018) & $45.33 \pm 5.06$ & $51.00 \pm 4.95$ & 0.002 & $44.25 \pm 4.78$ & $44.58 \pm 3.75$ & 0.076 \\
\hline Taher \& Parnow (2017) & Not provided & $51 \pm 8.31$ & NS & Not provided & $50 \pm 7.10$ & $<0.05$ \\
\hline Nawed, et al. (2018) & $37.44 \pm 14.86$ & $42.11 \pm 14.67$ & 0.01 & $37.70 \pm 6.42$ & $37.30 \pm 6.11$ & 0.741 \\
\hline \multicolumn{7}{|l|}{ 20m sprint (s) } \\
\hline Ayala, et al. (2016) & $3.3 \pm 0.18$ & $3.24 \pm 0.15$ & $<0.05$ & $3.28 \pm 0.15$ & $3.31 \pm 0.23$ & $<0.05$ \\
\hline Harøy, et al. (2017) & $2.83 \pm 0.08$ & $2.83 \pm 0.08$ & 0.71 & $2.83 \pm 0.09$ & $2.83 \pm 0.08$ & 0.68 \\
\hline Nawed, et al. (2018) & $3.47 \pm 0.16$ & $3.09 \pm 0.14$ & 0.001 & $2.48 \pm 0.10$ & $3.50 \pm 0.14$ & 0.695 \\
\hline \multicolumn{7}{|l|}{ Agility (s) } \\
\hline Ayala, et al. (2016) & $16.66 \pm 1.16$ & $16.26 \pm 0.83$ & NS & $16.59 \pm 0.98$ & $16.4 \pm 1.37$ & NS \\
\hline Taher \& Parnow (2017) & Not provided & $16.56 \pm 0.79$ & NS & Not provided & $16.53 \pm 1.64$ & NS \\
\hline \multicolumn{7}{|c|}{ Hip Adduction Strength (N.m/kg) } \\
\hline Harøy, et al. (2017) & $3.25 \pm 0.62$ & $3.51 \pm 0.63$ & $<0.001$ & $3.24 \pm 0.50$ & $3.22 \pm 0.35$ & 0.69 \\
\hline \multicolumn{7}{|c|}{ FMS total score (total score 21) } \\
\hline Rey, et al. (2018) & $13.90 \pm 2.21$ & $15.18 \pm 1.40$ & 0.046 & $14.81 \pm 1.40$ & $15.90 \pm 1.22$ & NS \\
\hline
\end{tabular}

\section{Discussion}

To our knowledge, there were a broad of information about the effectiveness of FIFA 11+ injury prevention program, which needed to be integrated. We focused on searching for evidences that FIFA 11+ program might help reduce injury rate and improve physical performance for male soccer players. There were few previous studies mentioned injury rate reduction and physical performance improvement of FIFA $11+$ program at the same time. A recent systematic review written by Sadigursky et al. had selected 6 articles out of 183 articles published between 2006 and 2016. They investigated 6344 soccer players of both sexes aged $>13$ years and found the FIFA $11+$ program reduced injuries by $30 \%$ in soccer players [14]. We tried to find not only reduction in injury rate, but also improvement in physical performance of male soccer players aged 16 years through FIFA 11+ program. In addition to previous information, we have successfully integrated new knowledge for recent 5 years of its efficacy in both perspectives. Multiple studies have proven that FIFA 11+ program could help reduce overall injury rate by stengthening core and lower extremity muscles and improving proprioception, balance, and coordination of the body $[7,9,15]$. Other studies also indicated that FIFA 11+ program might help improve physical performance of athletes such as, vertical jump, agility and hip adduction strength $[16,17]$.

There were a few studies that couldn't find significant differences between pre-post test after performing FIFA 11+ program on improving physical performance. An article written by Taher and Parnow found no significant differences between pro-post test compared to other static and dynamic stretching after performing FIFA 11+ program [16]. However, they mentioned the best way to explain their failure to find effects was lack of duration. Participants in their study only 
conducted the program for one day. FIFA $11+$ program has been proven to decrease the severe injuries by one half and overall injury rates by one third after over a few weeks of interventions [10]. Therefore, it might take longer time conducting FIFA $11+$ program to enhance motor capacity such as, core strength, balance and proprioception, balance and proprioception [18]. There was another study by Ayala, et al. found no improvements on agility, sprint time, and vertical jump after performing FIFA 11+ program in young elite soccer players (aged 16-18) [19]. However, another study written by Daneshjoo et al. [20] did find the improvements on vertical jump and $20 \mathrm{~m}$ sprint time after conducting the FIFA $11+$ program in young adults (aged 18.9 \pm 1.4 ), while Impellizeri et al. [21] found no improvements on $20 \mathrm{~m}$ sprint time, vertical jump and agility in adult players (aged 23.7 \pm 1.4 ). Therefore, Ayala, et al. [19] mentioned that differenct agegroup of participants could be a possible reason to explain discrepancy of reported results. Moreover, Compliance was an important factor to consider. One study written by Granelli, et al. had proven the degree of compliance was closely related to reduce severe injury rate in soccer [22]. Other than that there were a few studies mentioned about no effetiveness of FIFA $11+$ injury prevention program for futsal players [23,24]. Participants in Lopes' study found non-significant differences of physical performance improvement such as, balance and prorioception between intervention and control groups even with both short and long-term (10 weeks) intervention.

\section{Strength and Limitation}

The strength of this study was we researched recent studies for 5 years about FIFA 11+ program and selected statistically well-organized papers, which made it much worth trust. We also extracted proper information of FIFA 11+ injury prevention program and found evidences of its effectiveness to reduce injury rate and improve physical performance for male soccer players from each study. Since Meta-analysis results of included studies were not involved in this study, integrated statistical results need to be provided in the future study. Futhermore, this study only included data for male soccer players aged $\geq 16$; therefore data for female and effects of other sports will need to be furtherly researched. Third, information of obvious duration to perform FIFA 11+ program will need to be defined. Lastly, further research on various factors that FIFA 11+ injury prevention program does not affect futsal players' physical performance is needed.

\section{Conclusions}

In conclusion, we found that FIFA 11+ program showed significant reduction in injury rate and improvement in physical performance for male soccer players. These results may be due to the improvement of motor capacity. However, there was no immediate response to be effective; it at least took over a few weeks to determine significant differences between intervention and control groups. Therefore, performing FIFA $11+$ program might need to be considered by coaches and players as a warm-up protocol with a high compliance and correct methods to be effective.

\section{References}

1. Bizzini M, Junge A, Dvorak J. Implementation of the FIFA $11+$ football warm up program: how to approach and convince the Football associations to invest in prevention. Br J Sports Med. 2013; 47(12):803-806.

2. Faude $O$, Rößler R, Junge A. Football injuries in children and adolescent players: are there clues for prevention? Sports Med. 2013; 43(9):819-837.

3. Stubbe JH, van Beijsterveldt A-MM, van der Knaap S, et al. Injuries in professional male soccer players in the Netherlands: a prospective cohort study. J Athl Train. 2015; 50(2):211-216.

4. Grooms DR, Palmer T, Onate JA, Myer GD, Grindstaff T. Soccer-specific warm-up and lower extremity injury rates in collegiate male soccer players. J Athl Train. 2013; 48(6):782-789.

5. Trajković N, Gušić M, Molnar S, Mačak D, Madić DM, Bogataj Š. Short-Term FIFA 11+ Improves Agility and Jump Performance in Young Soccer Players. Int $J$ Environ Res Public Health. 2020; 17(6).

6. Silvers-Granelli H, Mandelbaum B, Adeniji O, et al. Efficacy of the FIFA $11+$ injury prevention program in the 
collegiate male soccer player. Am J Sports Med. 2015; 43(11):2628-2637.

7. Silvers-Granelli HJ, Bizzini M, Arundale A, Mandelbaum BR, Snyder-Mackler L. Does the FIFA 11+ Injury Prevention Program Reduce the Incidence of ACL Injury in Male Soccer Players? Clin Orthop Relat Res. 2017; 475(10):2447-2455.

8. (F-MARC) FMAaRC. The “11+” MANUAL A COMPLETE WARM-UP PROGRAMME TO PREVENT INJURIES. Secondary.

9. Al Attar WSA, Soomro N, Pappas E, Sinclair PJ, Sanders RH. Adding a post-training FIFA 11+ exercise program to the pre-training FIFA 11+ injury prevention program reduces injury rates among male amateur soccer players: a cluster-randomised trial. J Physiother. 2017; 63(4):235-242.

10. Chena M, Rodríguez ML, Bores AJ, Ramos-Campo DJ. Effects of a multifactorial injuries prevention program in young Spanish football players. J Sports Med Phys Fitness. 2019; 59(8):1353-1362.

11. Whalan M, Lovell R, Steele JR, Sampson JA. Rescheduling Part 2 of the $11+$ reduces injury burden and increases compliance in semi-professional football. Scand J Med Sci Sports. 2019; 29(12):1941-1951.

12. Ayala F, Calderón-López A, Delgado-Gosálbez JC, et al. Acute Effects of Three Neuromuscular Warm-Up Strategies on Several Physical Performance Measures in Football Players. PLoS One. 2017; 12(1):e0169660.

13. Parnow A. Level of functional capacities following soccerspecific warm-up methods among elite collegiate soccer players. J Sports Med Phys Fitness. 2016; 57(5):537-542.

14. Sadigursky D, Braid JA, De Lira DNL, Machado BAB, Carneiro RJF, Colavolpe PO. The FIFA 11+ injury prevention program for soccer players: a systematic review. BMC Sports Sci Med Rehabil. 2017; 9:18.

15. Nouni-Garcia R, Carratala-Munuera C, Orozco-Beltran D, Lopez-Pineda A, Asensio-Garcia MR, Gil-Guillen VF. Clinical benefit of the FIFA 11 programme for the prevention of hamstring and lateral ankle ligament injuries among amateur soccer players. Inj Prev. 2018; 24(2):149-154.

16. Vazini Taher A, Parnow A. Level of functional capacities following soccer-specific warm-up methods among elite collegiate soccer players. J Sports Med Phys Fitness. 2017; 57(5):537-542.

17. Akbari H, Sahebozamani M, Daneshjoo A, AmiriKhorasani M. Effect of the FIFA 11+ programme on vertical jump performance in elite male youth soccer players. Monten J Sports Sci Med. 2018; 7(2):17.

18. Gilchrist J, Mandelbaum BR, Melancon H, et al. A randomized controlled trial to prevent noncontact anterior cruciate ligament injury in female collegiate soccer players. Am J Sports Med. 2008; 36(8):1476-1483.

19. Pomares-Noguera C, Ayala F, Robles-Palazón FJ, et al. Training Effects of the FIFA 11+ Kids on Physical Performance in Youth Football Players: A Randomized Control Trial. Front Pediatr. 2018; 6:40.

20. Daneshjoo A, Mokhtar AH, Rahnama N, Yusof A. Effects of the $11+$ and Harmoknee Warm-up Programs on Physical Performance Measures in Professional Soccer Players. J Sports Sci Med. 2013; 12(3):489-496.

21. Impellizzeri FM, Bizzini M, Dvorak J, Pellegrini B, Schena F, Junge A. Physiological and performance responses to the FIFA $11+($ part 2): a randomised controlled trial on the training effects. J Sports Sci. 2013; 31(13):1491-1502.

22. Silvers-Granelli HJ, Bizzini M, Arundale A, Mandelbaum BR, Snyder-Mackler L. Higher compliance to a neuromuscular injury prevention program improves overall injury rate in male football players. Knee Surg Sports Traumatol Arthrosc. 2018; 26(7):1975-1983.

23. Lopes $M$, Lopes $S$, Patinha $T$, et al. Balance and proprioception responses to FIFA $11+$ in amateur futsal players: Short and long-term effects. J Sports Sci. 2019; 37(20):2300-2308.

24. Lopes M, Simões D, Rodrigues JM, Costa R, Oliveira J, Ribeiro F. The FIFA 11+ does not alter physical performance of amateur futsal players. J Sports Med Phys Fitness. 2019; 59(5):743-751. 